\title{
Simulating cosmic shear surveys with redshift information
}

\author{
Lindsay J. King ${ }^{1}$, Patrick Simon ${ }^{2}$ \\ and Peter Schneider ${ }^{2}$ \\ ${ }^{1}$ IoA, Cambridge University, Madingley Rd, Cambridge CB3 0HA, UK \\ email: ljk@ast.cam.ac.uk \\ ${ }^{2}$ IAEF Universitaet Bonn, Auf dem Huegel 71, D-53121 Bonn, Germany \\ email: psimon, peter@astro.uni-bonn.de
}

\begin{abstract}
Cosmic shear is an essential cosmological tool, breaking degeneracies inherent to CMB data and providing an independent check of cosmological parameters. Upcoming cosmic shear surveys with photometric redshift information will enable tighter constraints to be placed on cosmological parameters, and allow us to explore how dark matter evolves. A Monte Carlo method to rapidly simulate mock surveys enables us to estimate the covariance matrix for the shear correlation functions, and hence the expected errors on cosmological parameter estimates given survey specifications. We also make brief remarks on the separation of the cosmic shear signal from any due to intrinsic galaxy alignments.
\end{abstract}

\section{Introduction}

The large-scale structure (LSS) of the Universe causes deflection and distortion of propagating light bundles. This cosmic shear signal is manifest in the distorted, correlated, shapes of distant galaxies. The statistical properties of the ellipticities of these galaxies depend on the (dark) matter power spectrum and cosmological parameters, thus providing access to these quantities.

In the aftermath of WMAP, why should we bother with cosmic shear surveys? Cosmic shear surveys provide an independent probe of cosmological parameters, both providing cross-checks and breaking degeneracies inherent to CMB data. Of particular note is the near-orthogonality of constraints in the $\Omega_{\mathrm{m}}-\sigma_{8}$ plane (e.g. van Waerbeke et al. 2002), where $\Omega_{\mathrm{m}}$ is the matter density parameter and $\sigma_{8}$ is the power spectrum normalisation.

Cosmic shear surveys with redshift information enable us to study the evolution of dark matter and to place tighter constraints on cosmological parameters. Here, we focus on the improvement in constraints on cosmological parameters coming from two-point correlations of galaxy ellipticities, using a Monte Carlo method to rapidly simulate mock surveys. See contributions in this volume from David Bacon, Alan Heavens and Andy Taylor. For full details of the technique used for the numerical simulations, see the contribution from Patrick Simon, and also Simon, King \& Schneider (2004) for further details of this work.

\section{Comparison of $\mathrm{CMB}$ and lensing degeneracies}

For the CMB power spectrum, Jungman et al. (1996) derived an expression for the Fisher information matrix which yields the attainable accuracy on cosmological parameters; it is the derivatives of the CMB power spectrum with respect to parameters which 


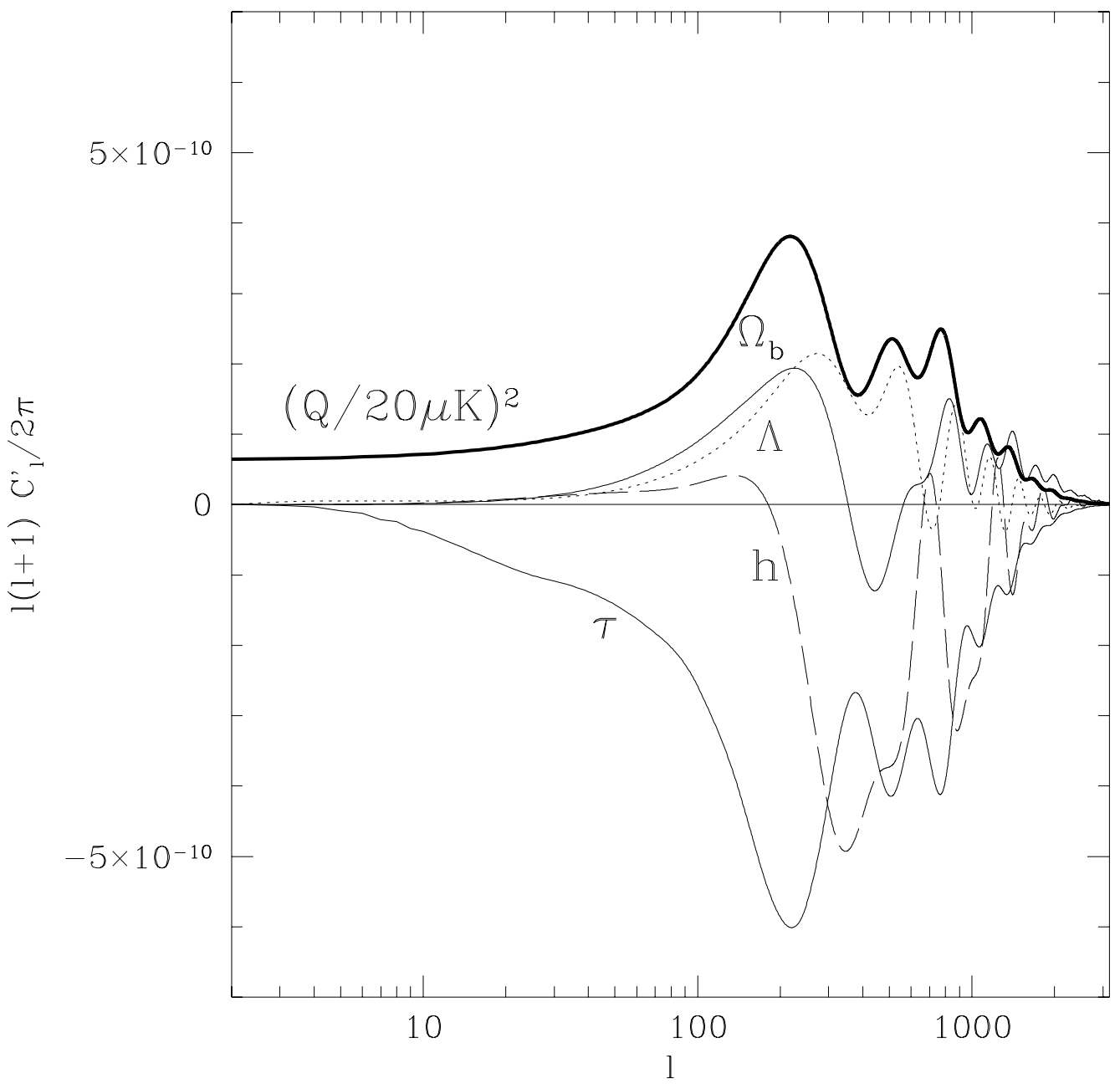

Figure 1. Derivatives of the CMB power spectrum with respect to various cosmological parameters: baryon density parameter $\Omega_{\mathrm{b}}$, cosmological constant $\Lambda$, Hubble parameter $h$, optical depth due to reionization $\tau$ and quadrupole normalization $Q$. From Tegmark, Taylor \& Heavens (1997).

determine the errors. Figure 1 (from Tegmark, Taylor \& Heavens 1997) shows the derivatives of the CMB power spectrum as a function of $l$ for baryon density parameter $\Omega_{\mathrm{b}}$, cosmological constant $\Lambda$, Hubble parameter $h$, optical depth due to reionization $\tau$ and quadrupole normalization $Q$, as described in that paper.

Analogously for lensing, Figure 2 (from King \& Schneider 2003) shows the derivatives of the redshift averaged lensing two-point shear correlation function $\xi_{+}$with respect to $\Omega_{\mathrm{m}}, \sigma_{8}$, power spectrum shape parameter $\Gamma$ and power spectrum spectral index $n$.

These figures illustrate the extent to which various parameters are degenerate; the more degenerate the parameters, the more similar the form of the derivatives curves. Note that the CMB curves have more structure than the lensing ones. 


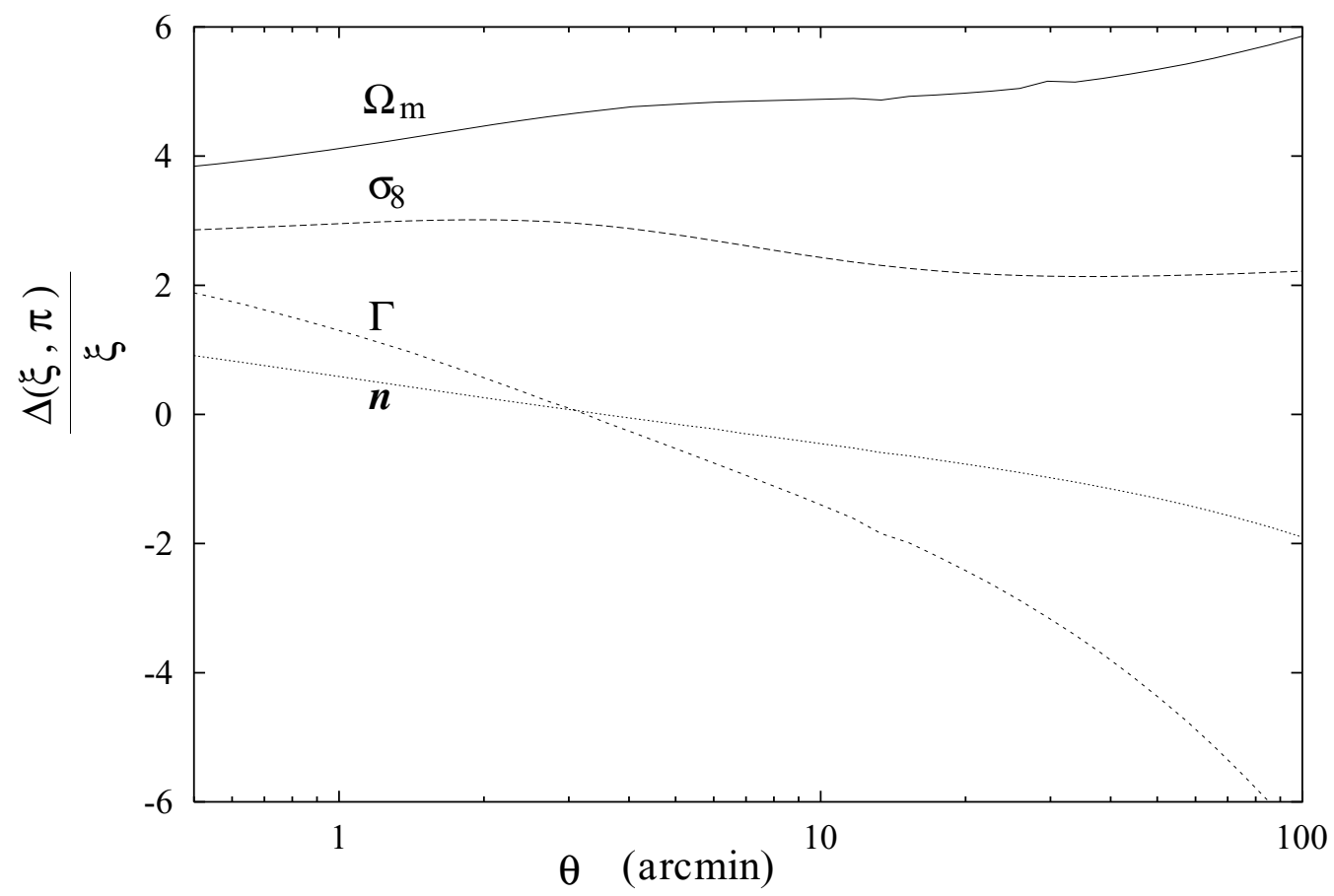

Figure 2. Derivatives of the two-point shear correlation function with respect to various cosmological parameters: $\Omega_{\mathrm{m}}$ is the matter density parameter, $\sigma_{8}$ is the power spectrum normalisation, $\Gamma$ is the shape parameter and $n$ is the spectral index. From King \& Schneider (2003).

\section{How much does redshift information help parameter constraint?}

$\mathrm{Hu}$ (1999) investigated the extent to which crude redshift information decreases the errors on parameters, using the convergence power spectrum as the vehicle of cosmological information. He found that errors on parameters, in particular those that govern the rate of growth of structure, can be decreased by up to an order of magnitude, within the adiabatic cold dark matter class of models. Here, we use shear correlation functions binned in redshift, and in addition allow for cross-correlations between measurements of the shear signal at different angular scales.

\subsection{Quantifying cosmic shear: the two-point shear correlation functions}

The degree to which galaxy ellipticities are correlated gives a measure of the strength of cosmic shear. We concentrate on two-point shear correlation functions, $\xi_{ \pm}(\theta)$, since their evaluation is independent of any gaps in the data field; other statistics can be derived from these. In the absence of intrinsic correlations

$$
\begin{aligned}
& \xi_{ \pm}(\theta)=\left\langle\gamma_{\mathrm{t}} \gamma_{\mathrm{t}}\right\rangle \pm\left\langle\gamma_{\times} \gamma_{\times}\right\rangle(\theta) \\
& \xi_{\times}(\theta)=\left\langle\gamma_{\mathrm{t}} \gamma_{\times}\right\rangle(\theta)
\end{aligned}
$$

where $\theta$ is the angular scale, $\gamma_{t, \times}$ are the tangential and cross components of the shear, and the angle brackets denote averaging over all pairs within the separation bin defined by $\theta$. 
The two-point shear correlation functions may also be expressed as

$$
\xi_{ \pm}(\theta)=\int_{0}^{\infty} \frac{\mathrm{d} l l}{2 \pi} \mathrm{J}_{0,4}(l \theta) P_{\kappa}(l)
$$

where $P_{\kappa}(l)$ is the effective convergence power spectrum as a function of angular wavevector $l$. In other words, the observable two-point correlation functions are filtered versions of the convergence, or equivalently shear, power spectrum.

From now on we focus on $\xi_{+}$which contains most of the relevant cosmological information on scales typically of interest. For the case where specific redshifts are available, consider sources on sheets at $z_{i}$ and $z_{j}$; the correlation function is then measured as a function of not only separation, but also redshift

$$
\begin{array}{r}
\xi_{+}\left(\theta, z_{i}, z_{j}\right)=\left\langle\gamma\left(\phi, z_{i}\right) \gamma^{*}\left(\phi+\theta, z_{j}\right)\right\rangle= \\
\frac{9 H_{0}^{4} \Omega_{\mathrm{m}}^{2}}{4 c^{4}} \int_{0}^{\min \left[w_{i}, w_{j}\right]} \frac{\mathrm{d} w}{a^{2}(w)} \times R\left(w, w_{i}\right) R\left(w, w_{j}\right) \\
\times \int \frac{\mathrm{d} l l}{(2 \pi)} P_{\delta}\left(\frac{l}{f(w)}, w\right) \mathrm{J}_{0}(l \theta),
\end{array}
$$

where $a$ is the scale factor normalised so that $a=1$ today. $w_{i}$ is the comoving distance at $z_{i}, f(w)$ is the comoving angular diameter distance, depending on spatial curvature $K$, and $R\left(w, w^{\prime}\right)=f\left(w^{\prime}-w\right) / f\left(w^{\prime}\right)$.

The final integration is over the 3 -D power spectrum. The first integral contains geometrical factors and the scale factor, and is performed out to the minimum of the two comoving distances, since only structure common to light travelling from the two sheets induces correlations. From this we see that if redshift information is available, we can probe the evolution of dark matter.

We assume from now on that we measure the correlation functions in angular separation and redshift bins.

\section{Ingredients for cosmological parameter constraint}

The log-likelihood function for cosmological parameter estimates is distributed as $\chi^{2} / 2$ so that

$$
\chi^{2}(\pi)=\sum_{i j}\left(\xi^{\mathrm{t}}-\xi(\pi)\right)_{i}\left[\mathbf{C}^{-1}\right]_{i j}\left(\xi^{\mathrm{t}}-\xi(\pi)\right)_{j}
$$

where $\pi$ are the parameters under consideration, $\mathrm{t}$ denotes the fiducial model, $\mathbf{C}$ is the covariance matrix and $i, j$ denote the bins between which the covariance is determined. We determine the covariance matrix using a Monte Carlo technique described in Simon, King \& Schneider (2004). The covariance matrix is given by

$$
\mathbf{C}_{i j}=\left\langle(\hat{\xi}-\langle\hat{\xi}\rangle)_{i}(\hat{\xi}-\langle\hat{\xi}\rangle)_{j}\right\rangle_{N_{f}},
$$

where the outer average is performed over the realisations (Universes) and the inner averages over the fields included from each of those realisations.

The auto- and cross-correlation functions are determined as a function of angular separation for each redshift bin available. Figure 3 outlines the process of parameter constraint. A population of sources is lensed and the redshift binned auto- and crosscorrelation functions are determined over a number of fields for each "Universe". The 


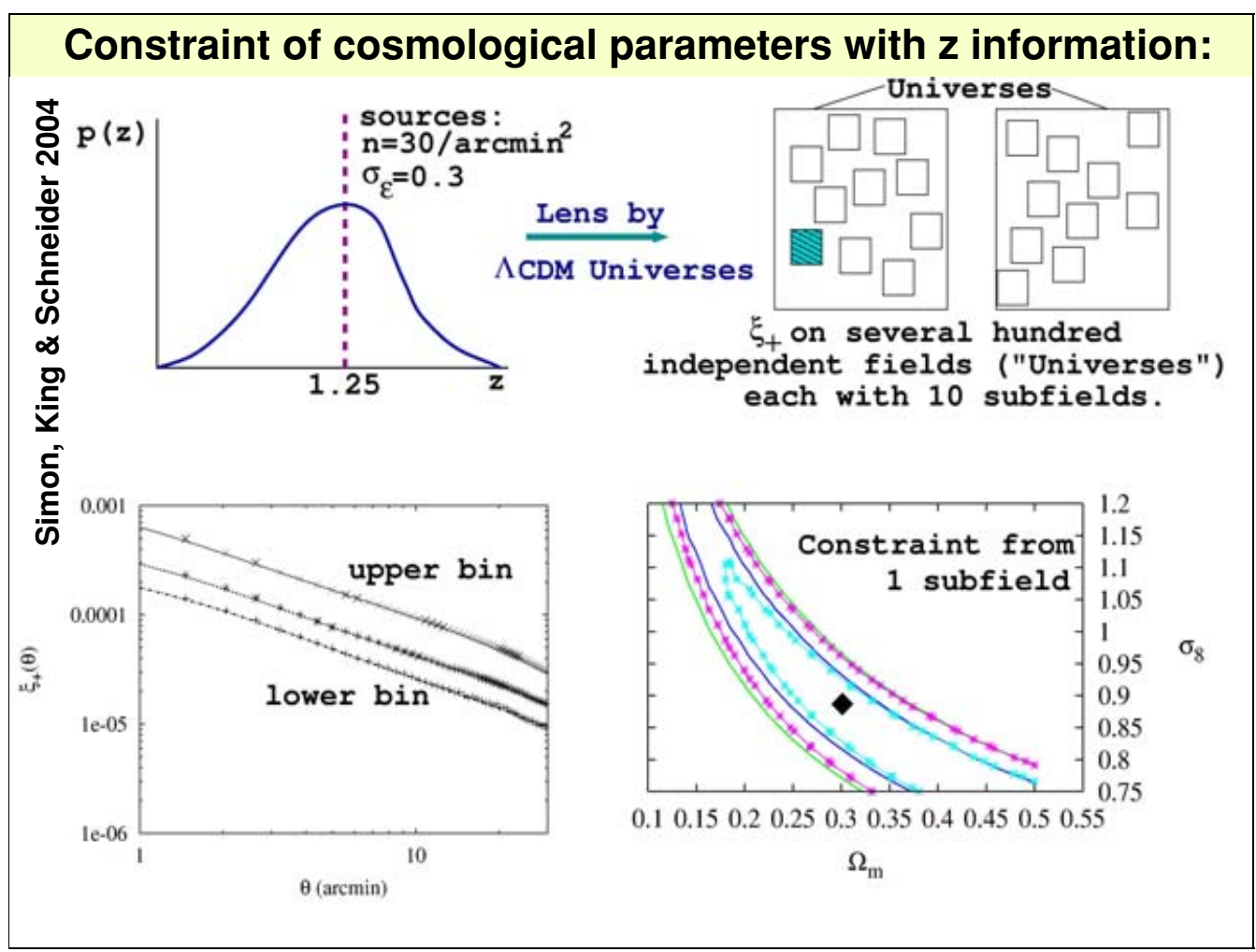

Figure 3. Cartoon illustrating the process of cosmological parameter constraint. See text for further details.

averages of these correlation functions agree well with theoretical expectations. The lower right panel shows the constraint from one sub-field in the $\Omega_{\mathrm{m}}-\sigma_{8}$ plane, without (solid lines) and with (lines with superimposed symbols) redshift information. The fiducial model is marked with a diamond.

We have explored constraints on parameters when 2, 3 or 4 redshift bins are used. For large numbers of redshift bins, it is convenient to perform a Fisher analysis. We find that with no priors, parameter estimates improve by a factor of between 4-8 going to 2 redshift bins, and a factor of 5-10 going to 4 redshift bins. As in $\mathrm{Hu}$ (1999) we find that parameters determining the rate of growth of structure are most favourably affected by binning.

Figure 4 shows the Fisher error for 2, 3 and 4 bins as a percentage of the error for no binning, for each of $\Omega_{\mathrm{m}}, \Omega_{\Lambda}$ and $\Gamma$.

\section{Caveats and questions}

As discussed in Simon, King \& Schneider (2004), the assumption of gaussianity causes the covariance to be underestimated at small scales (e.g. Scoccimarro, Zaldarriaga \& Hui 1999). However, on linear scales, or for investigating which survey strategy is optimal for a particular purpose, our method performs very well. For analysis of a real survey, 


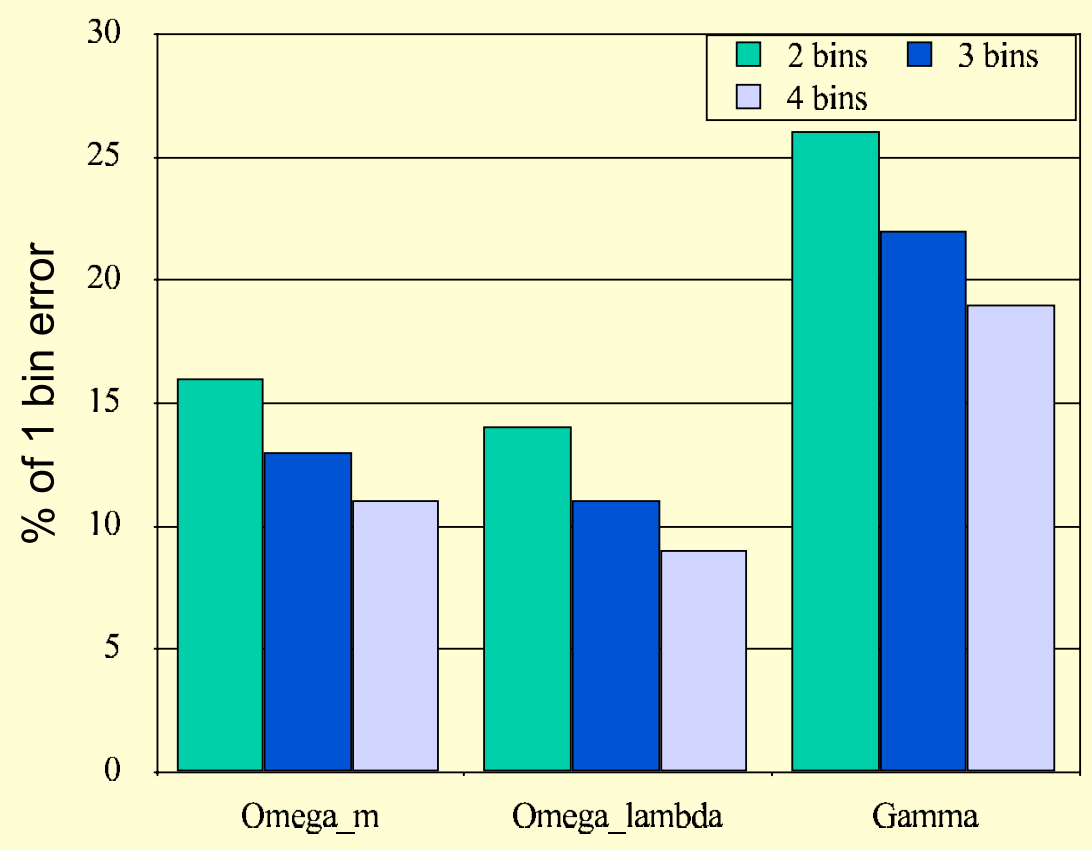

Figure 4. Extent to which parameter errors are reduced with redshift binning, shown as a percentage of the error with no redshift binning (1 bin error).

one would use (much more time consuming) ray tracing through high resolution N-body simulations to obtain covariance matrices for particular cosmologies.

Another issue which has been raised in the past few years is that of intrinsic galaxy alignments:

\subsection{Do intrinsic galaxy alignments mess up cosmic shear?}

Intrinsic galaxy alignment (IA) has often been neglected in cosmic shear surveys, but it is poorly understood. In the limit of very weak lensing $\epsilon=\epsilon^{(\mathrm{s})}+\gamma$. Usually, it is assumed that shape distortion arises solely from lensing. But, if intrinsic alignments in source ellipticities, arising at the epoch of galaxy formation, also play a role then $\xi_{+}$must be written as

$$
\xi_{+}=\left\langle\epsilon_{i} \epsilon_{j}^{*}\right\rangle=\left\langle\gamma_{i} \gamma_{j}^{*}\right\rangle+\underbrace{\left\langle\epsilon_{i}^{(\mathrm{s})} \epsilon_{j}^{(\mathrm{s}) *}\right\rangle}_{\text {intrinsic }}=\xi_{+}^{\text {lens }}+\underbrace{\xi_{+}^{\mathrm{int}}}_{\text {intrinsic }},
$$

and similarly for $\xi_{-}$. In other words, an intrinsic signal "contaminates" the lensing signal. If cosmic shear surveys are to be used to place constraints on cosmological parameters and on the matter power spectrum, then any intrinsic signal must be accounted for in the analysis.

Physically close galaxies form in similar gravitational fields, and thus might be expected to have correlations in their shapes. A couple of mechanisms which may give rise to such an effect are 
- Tidal stretching - where the ellipticity of a (luminous) galaxy is determined by the shape of the (dark matter) halo in which it forms. This is most relevant for elliptical galaxies, and the ellipticity is linear in the tidal field.

- Tidal torquing during galaxy formation may give rise to correlations in angular momenta (spins) and projected ellipticities of close galaxies. Hoyle (1949) suggested that a galaxy acquires angular momentum by tidal torques from matter surrounding its protogalaxy. This mechanism is most relevant for spiral galaxies.

Numerical and analytic predictions for the magnitude of the effect span more than an order of magnitude; hence it is fair to say that it is poorly understood. One possibility to deal with any potential IA is to down-weight physically close galaxy pairs (King \& Schneider 2002; Heymans \& Heavens 2003). A better solution is to perform shear correlation function tomography - this allows separation of IA and cosmic shear signals without assuming an IA model - we obtain constraints on IA and a clean cosmic shear signal (King \& Schneider 2003). Recently, Hirata \& Seljak (2004) have proposed that a cross-term between IA and cosmic shear may be important; along a line of sight where there is a high IA signal, cosmic shear of higher redshift sources can also be boosted due to the tidal field giving rise to the IA signal. Depending on the origin of IA, this cross-term can be zero or be of concern in surveys.

\section{Conclusions}

Cosmic shear can be used to study the evolution of the large scale structure in the Universe; it benefits from source redshift estimates. We can rapidly simulate cosmic shear surveys to obtain covariance matrices, an essential ingredient in parameter estimation. As an application we considered to what extent shear correlation functions enable tighter constraints to be placed on cosmological parameters when even crude redshift bins are used. The errors on parameters can be decreased significantly using redshift binning.

Intrinsic galaxy alignment may be an important consideration, though the origin of this effect is not understood and the magnitude very uncertain. Shear correlation function tomography could be used to constrain intrinsic galaxy alignment and to obtain a clean lensing signal. A caveat to this was recently proposed by Hirata \& Seljak (2004) - a crossterm between IA and cosmic shear. Assuming that we can deal with IA, the subsequent combination of cosmic shear survey results with other tracers of LSS, with CMB lensing and with the primary and secondary anisotropies of the CMB itself will be incredibly powerful in addressing central issues in cosmology: how structures form and evolve.

\section{Acknowledgements}

For financial support, we would like to thank the Royal Society (LJK), the Deutsche Forschungsgemeinschaft under the projects SCHN 342/3-1 and Graduiertenkolleg 787, and the German Ministry for Science and Education (BMBF) through the DLR under project 50 OR 0106.

\section{References}

Heymans, C. \& Heavens, A. 2003, MNRAS 339 711-720

Hirata, C. \& Seljak, U. 2004, astro-ph/0406275

Hoyle, F. 1949, in Problems of Cosmical Aerodynamics (Dayton : Central Air Documents Office), ed. J. M. Burgers \& H. C. van de Hulst, 195

Hu, W. 1999, ApJL 522 L21-L24

Jungman, G., Kamionkowski, M., Kosowsky, A. \& Spergel, D. 1996, Phys. Rev. Lett. 76, 10071010 
King, L. \& Schneider, P. 2002, A\&A 396 411-418

King, L. \& Schneider, P. 2003, A\&A 398 23-30

Scoccimarro, R., Zaldarriaga, M. \& Hui, L. 1999, ApJ 527, 1-15

Simon, P., King, L.J. \& Schneider, P. 2004, A\&A 417 873-885

Tegmark, M., Taylor, A. \& Heavens, A. 1997, ApJ 480 22-35

van Waerbeke, L. et al. 2002, A\&SA 393 369-379 